\title{
Sobre la función del edificio singular de Cancho Roano (Zalamea de la Serena, Badajoz)
}

\author{
About the Function of the Singular Building of Cancho Roano \\ (Zalamea de la Serena, Badajoz)
}

\author{
Fernando LóPEZ PARDO \\ Publicado por primera vez en Gerión 8, 1990, 141-162
}

En Cancho Roano, en el valle del Ortiga, afluente del Guadiana, Joan Maluquer sacó a la luz una construcción imponente. La "rareza" del monumento en tal contexto ha suscitado un gran interrogante dentro de la Protohistoria del Sudoeste peninsular. Tanto es así que el monumento de Cancho Roano, como documento histórico, ha sido utilizado con mucha precaución. Las veces que se ha tenido en cuenta. En este trabajo pretendemos, en la línea de su excavador, hacer dos propuestas de interpretación sobre el uso original del edificio.

En una descripción muy somera, se puede destacar que se trata de una construcción de adobe rodeada por un gran muro de piedra de dos metros de ancho y dos de altura, de aparejo muy cuidado, especialmente en las esquinas, donde hay bloques ciclópeos en las hiladas inferiores. La forma cuadrada de la construcción queda rota por un patio exterior en el lado de levante, que era la zona de acogida y de acceso. Dicho patio estaba enlosado con pizarra, y un banco corrido se extendía al pie de sus muros. ${ }^{1}$

La cámara principal de la estructura es una habitación ciega, muy amplia, con un pilar de adobe en el centro que sostenía la techumbre. Al norte de esta habitación central existe, según su excavador, J. Maluquer, una zona residencial compuesta por una larga nave exterior y tres habitaciones comunicadas entre sí. En el lado sur una zona de almacenamiento que se compone de una nave y dos estancias más. Aquí se encontraron apiladas una veintena de ánforas globulares fenicias. La mayoría de las entradas son por eje acodado, incluido el acceso al edificio que se realizaba por uno de los costados del patio (fig. 1).

Delante de todo ello se extiende una nave de más de tres metros de ancho que va de un extremo al otro el monumento, donde hay dos cuerpos salientes que dan forma al patio abierto de la entrada. En el lado norte se han podido reconocer dos escaleras. Una da entrada al edificio, pues todas las estancias están sobreelevadas 1,40 metros, menos la cámara central, que se mantiene al mismo nivel del patio. La otra escalera llevaba a las cubiertas superiores. Estas eran planas, en forma de azotea, según la verosímil reconstrucción propuesta en la segunda memoria de excavación (fig. 2). ${ }^{2}$

\footnotetext{
1 La descripción la hemos tomado de las dos memorias de excavación del yacimiento que han aparecido hasta el presente: MALUQUER 1981 e ID. 1983.

2 Maluquer 1983, 140.
} 


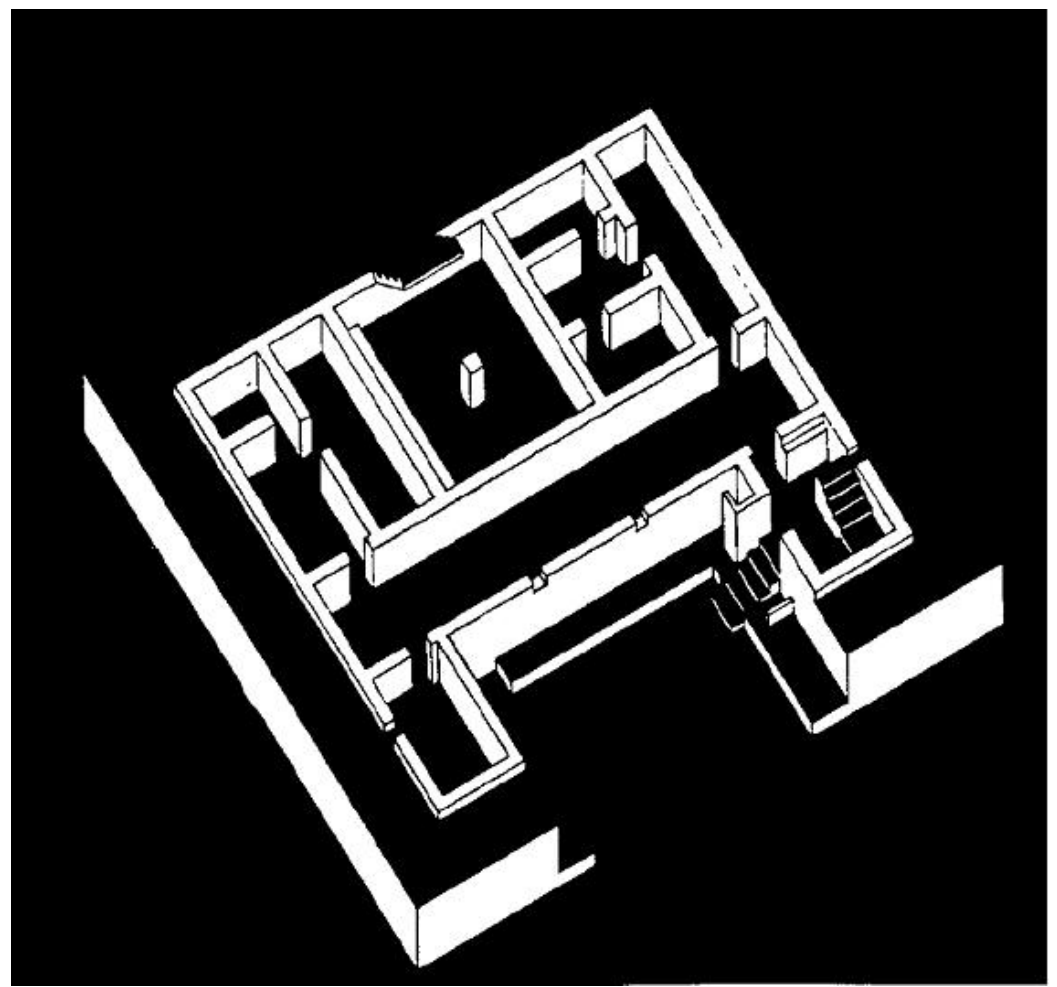

Fig 1. Planta analítica del monumento de Cancha Roano. C.R. II. 134, fig. 63.

La construcción se fecha en el siglo VI a.C., y cuenta como elementos de datación un conjunto de ánforas halladas in situ y un recipiente procedente de Naucratis. En un momento indeterminado del siglo $\mathrm{V}$ a.C. el edificio sufre un incendio y se destruyen algunos muebles y enseres, así como el depósito de ánforas del lado sur. También caen algunas partes de la techumbre. Sin embargo, la azotea central se mantiene y a fines del siglo y hasta comienzos del IV a.C., quizá durante una veintena de años, se utilizará como ustrinum elevado, donde se quemarán los cadáveres con destino a una necrópolis de urnas que comienza a desarrollarse en su entorno. Los carbones y cenizas con los restos de ajuares se barrerán hacia las estancias situadas justo debajo, a través de los techos hundidos. ${ }^{3}$

3 J. Maluquer, a través de la abundante información arqueológica, reconstruye de la siguiente manera la última utilización de este complejo: "los escombros de las incineraciones... se barrieron sobre los huecos de las naves y habitaciones hundidas. rellenándolas poco a poco. Se echaban allí los carbones humeantes junto con la cerámica utilizada en las libaciones que habían sido arrojadas ritualmente en la hoguera, más los enseres o envases de los alimentos consumidos probablemente en un gran banquete fúnebre celebrado durante la propia incineración. Para apagar estos restos, barridos hacia el fondo de las antiguas estancias, los cuidadores del ritual apagaban. mediante espuertas de tierra, los carbones encendidos, ocasionando el típico aspecto de 


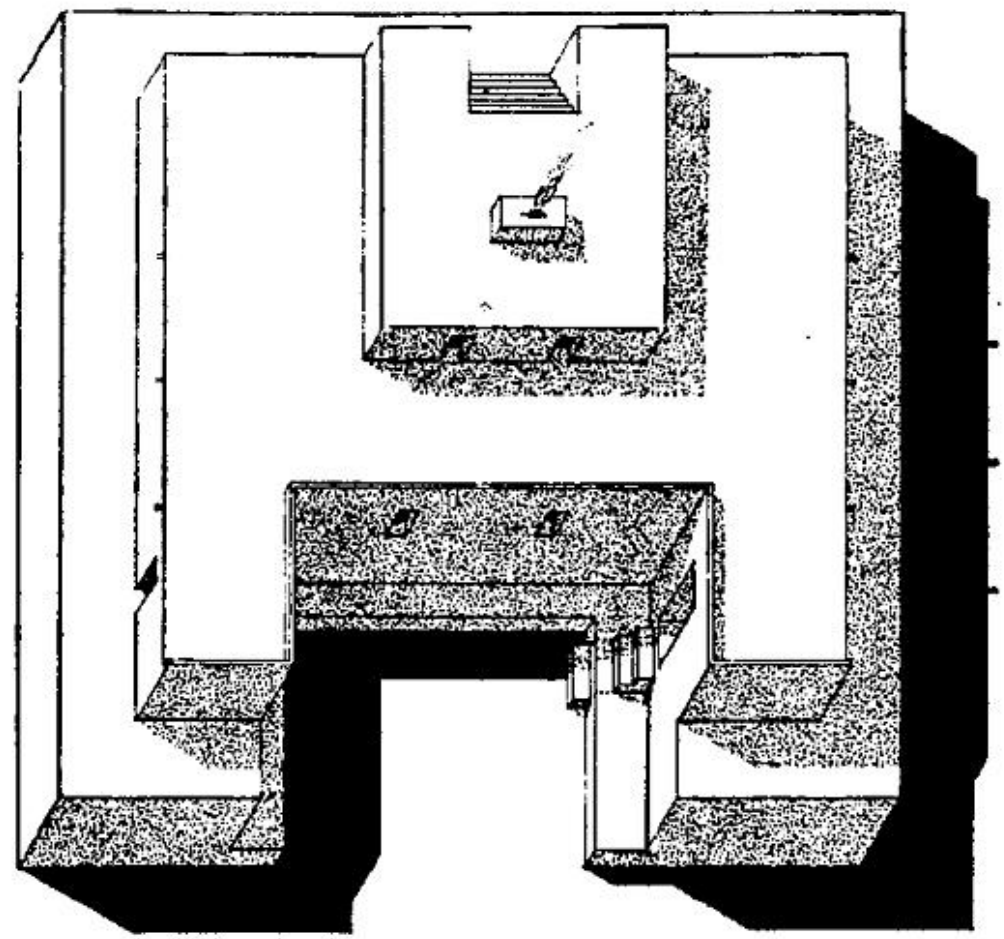

Fig. 2. Restitución analítica de las cubiertas del monumento de Cancho Roano. C.R. II, 135, fig. 64 .

De esta fase es la considerable cantidad de cerámica griega, la mayoría copas - kylikes - de barniz negro con labio exterior y sin decorar, y de figuras rojas de factura mediocre. Sin duda son de los inicios del siglo IV a.C. Ningún resto arqueológico va más allá de esta época. ${ }^{4}$

La importancia de este magnífico hallazgo arqueológico y su rareza en el entorno en que se encuentra obligó a J. Maluquer a analizar detenidamente todas las posibilidades sobre su oscuro origen. Llegó a plantear, pues, tres hipótesis de trabajo: la primera, que el monumento tuviera un modelo indígena, derivado de la arquitectura megalítica occidental; la segunda, un origen continental de grupos "norte pirenaicos" de la Edad del Bronce. Y como última hipótesis, que tuvieran un modelo oriental, importado por comerciantes mediterráneos.

En la primera memoria de excavación, J. Maluquer ya desestima las dos primeras posibilidades por inconsistentes. No encontró en la tradición autóctona nada anterior

\footnotetext{
lentejones disimétricos intercalados en las cenizas que se acusan en la misma sección general" (MaLuQuer 1981, 275).

4 Maluquer 1981, 276-277.
} 
que se le pareciera, ni tampoco más allá de los Pirineos había construcciones similares. Optó, por lo tanto, por atribuirle una raíz oriental.

Tras la primera memoria publicada sobre el yacimiento, A. Blanco, en un artículo en una revista de divulgación, no acepta tal origen, fundiendo en uno el uso final del edificio y su función inicial. Pretendiendo, en suma, su parecido con los amontonamientos de tierra y ceniza con un ara de ofrendas encima, que son los "brandgraber" de origen centroeuropeo.

En el segundo volumen sobre la excavación, su autor desecha tal paralelo para la construcción del edificio. Según él, estas referencias en todo caso no conciernen al propio edificio, sino a la masa de cenizas y carbones que lo amortizaron. Señalando, por otra parte, que aunque en algunos "brandgraber" existen construcciones subyacentes, de la bibliografía se desprende que nada conocido puede paralelizarse ni siquiera remotamente con el edificio extremeño. ${ }^{5}$

En definitiva, se reafirma J. Maluquer en que los paralelos se hallan en el Mediterráneo Oriental. Se trataría de un santuario comparable a los templos de tipo migdal, con antecedentes en el segundo milenio en Meggido y Sechem. La distribución, de habitaciones en torno a otra más amplia le recuerdan los templos de planta cuadrada de Tananir en Palestina y el de Ammán. Reproduciendo su distribución los elementos esenciales de un palacio oriental, ya que el templo es considerado como casa del dios en la tierra. De ahí el gran parecido que encuentra entre el santuario de Cancho Roano y el palacio de Tiglathpiliser III en Arlan Tash y otros hilani de Siria. ${ }^{6}$

A pesar de este parecido, J. Maluquer considera que la aparición de este tipo de planta en Cancho Roano no se ha producido por transmisión directa oriental hacia Occidente. Sino a través del mundo griego, que tenía sus puntos de contacto en Rodas, Chipre, Ugarit, Tell Sukkas y muy especialmente, según remarca el autor, en Al Mina.

Precisamente es en Al Mina, en el bajo Orontes, donde encuentra unos paralelos, que califica de "sorprendentes", en los edificios del barrio comercial. ${ }^{7}$ Esta semejanza se destaca sobre todo en la forma de U de la planta de los almacenes de Al Mina y del edificio de Cancho Roano.

Dicha similitud y los abundantes fragmentos de copas áticas, que él mismo reconoce que no corresponden a la utilización del edificio como tal, le hacen decantarse por la idea de que son los griegos los que han hecho construir este santuario en Cancho Roano, eso sí, reconociendo el origen semita del modelo. ${ }^{8}$

La construcción estaría en consonancia con un cambio paulatino de la organización del gran comercio en Occidente, que tras el predominio de los canales de distribución fenicios se pasaría a un predominio griego temprano. Desgraciadamente, como el mismo autor afirma, la sustitución de un predominio por otro no se produce

\footnotetext{
5 Blanco 1982, 42-43; Maluquer 1983, 130.

6 Maluquer 1981, 284.

7 Maluquer 1983, 132; Wooley 1938, 1-30.

8 El autor ve como inverosímil que sea obra de un indígena muy helenizado o semitizado (MALUQUER 1981, 283-284).
} 
hasta el siglo V a.C. y por tanto no coincide cronológicamente con la construcción del monumento, que es anterior. ${ }^{9}$

Concluye diciendo: “... ese modelo conocido y utilizado por los comerciantes griegos y con seguridad por los rodios tiene su expansión por el Mediterráneo en época incierta que acompaña en parte a la actividad griega, por lo cual es muy probable que pueda detectarse en zonas de gran presencia griega de la Península y probablemente en la costa levantina y del sudeste". ${ }^{10}$

Tras el incendio del edificio, éste siguió teniendo una función sacra, según el autor, esta vez sólo de ara o de ustrinum sobre los escombros (sobre ello no se decanta J. Maluquer). Piensa que o bien sufrió un cambio de rito, o se trataba de un intento voluntario por destruir el santuario. En principio, considera que la aparición de varios enterramientos de cremación en el entorno del edificio significa simplemente un cambio de ritual o un cambio de advocación. Ello le llevó a considerar también que la divinidad que allí recibía culto era de carácter funerario. ${ }^{11}$

La interpretación de J. Maluquer, generalmente bien fundada, es enormemente sugerente y en gran parte asumible.

No obstante, creemos que el excavador se ha visto lógicamente condicionado por la evidencia material hallada, que corresponde abrumadoramente a la última ocupación, y trasladó por ello parte de las conclusiones referidas a esa época a la fase inicial. Así, por un lado, no creyó en un cambio de uso del edificio, y por tanto supuso que si al final tuvo un uso religioso, éste sería extensible a la construcción y a la primera fase de utilización. Por otro lado, al aparecer abundante cerámica griega, también en la época final, serían los griegos quienes implantaron este modelo de edificio, aunque reconocía muy acertadamente su origen próximo-oriental.

El nexo de transmisión del mundo semita al griego de este tipo de edificio lo encontraba ya en la costa siria-palestina. En los almacenes de Al Mina.

Sin embargo, el paralelismo con los almacenes de Al Mina no puede ser aducido como argumento de la transmisión griega. Como acaba de demostrar A. J. Graham, la consideración de Al Mina como una colonia griega es incorrecta, pues se hizo creyendo que era la antigua Posideum, que se encuentra en realidad más al sur. Tanto el material recogido, así como la arquitectura de Al Mina atestiguan, según este autor, que no es una ciudad griega, sino que probablemente fue fundada por los fenicios. A lo que añade que la presencia de abundante cerámica griega pintada es evidencia del comercio y no de la ocupación. El escaso número de graffiti sugiere la existencia de algunos residentes griegos, pero en la misma medida que en otros lugares del Este mediterráneo. ${ }^{12}$ Por tanto, los hallazgos de Al Mina no confirman la mediación griega en la transmisión del modelo al Extremo Occidente.

Precisamente en la región siria y sus proximidades se encuentran edificios de uso principesco que reproducen también la planta palacial básica, sin poder considerarlos estrictamente como tales, son los Bît-hilani.

9 Maluquer 1983, 136-137.

10 Maluquer 1983, 136.

11 Maluquer 1983, 140-141.

12 Graham 1983, 51-65; sobre los materiales no griegos del yacimiento, véase: Plat TAYlor 1956, 62-92. 


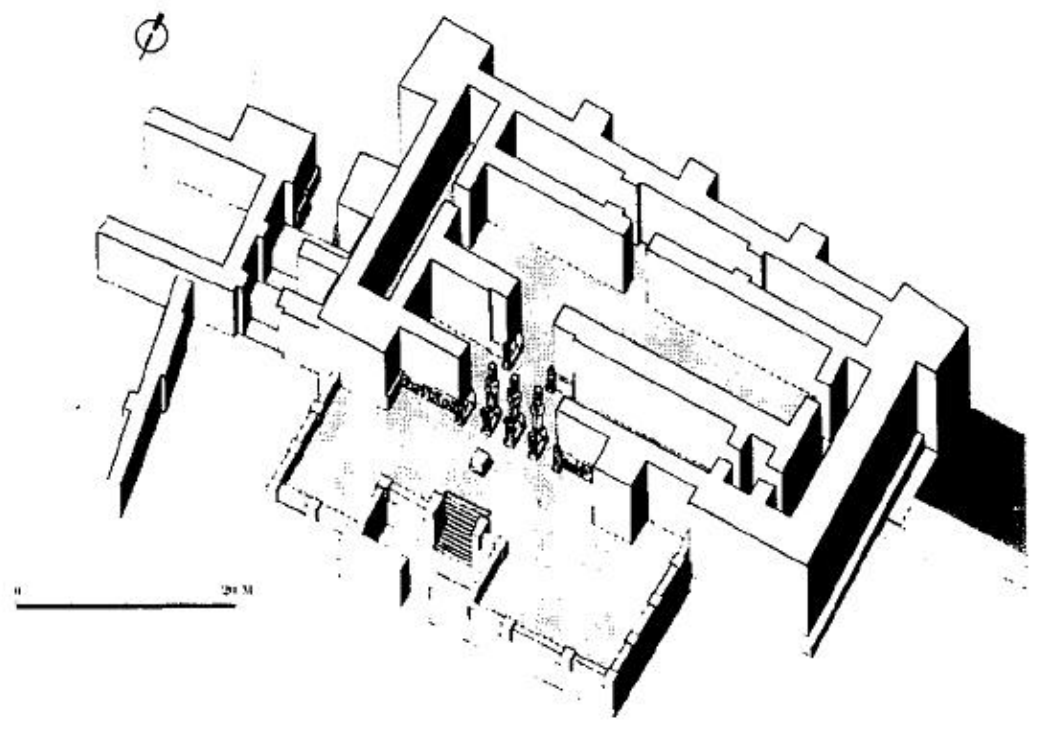

Fig. 3. Tell-Halaf. Bit-hilani de Kapara. S. VIII a.C. Según P. Amiet: L'Art antique du Proche- Orient, Paris, 1977, 477.

Su uso no está claro, ya que no cuentan con suficientes habitaciones como para ser habitados permanentemente por el príncipe. No pueden ser considerados, por tanto, como palacios al uso, Probablemente, por su posición ante una explanada o plataforma amplia, servían para actos de representación del monarca.

Un enorme paralelismo encontramos entre el edificio de Zalamea de la Serena y los hilaní del siglo VIII a.C. de Tell Taynat y TeIl Halaf. Todos ellos tienen un aspecto macizo, cuentan con un patio de entrada abierto, habitaciones alargadas, etc. ${ }^{13}$ (figs 3 у 4$)$.

Sin embargo, existe una diferencia notable que desaconseja interpretar que el edificio de Cancho Roano tuvo una función similar a las construcciones de Siria, aunque mantengamos la creencia de que son su paralelo más exacto. El edificio extremeño cuenta con una cámara central in profundis no abierta a las otras habitaciones, que parece ser el centro de todo el edificio, cámara y articulación que no aparecen en los Hilani (fig. 1).

En resumen, dentro de estos dos supuestos, templo o edificio palacial, la diferenciación es difícil de establecer a partir de los modelos, ya que la forma y organización interna de muchos santuarios próximo-orientales, tanto del segundo como del primer milenio, imitan la forma palacial básica de la zona. ${ }^{14}$

13 Amiet 1977, 477 y 483.

14 Muchos templos como el de Ninni-Zaza en Mari, del 1700 a.C. fueron construidos a semejanza del área de representación del palacio. El patio interior servía en el templo para actos de culto y la habitación de aparato palacial que abre sobre el patio es la cella. PARrot 1967, 34; los santuarios de Melkart de Tiro y de Yaveh en 


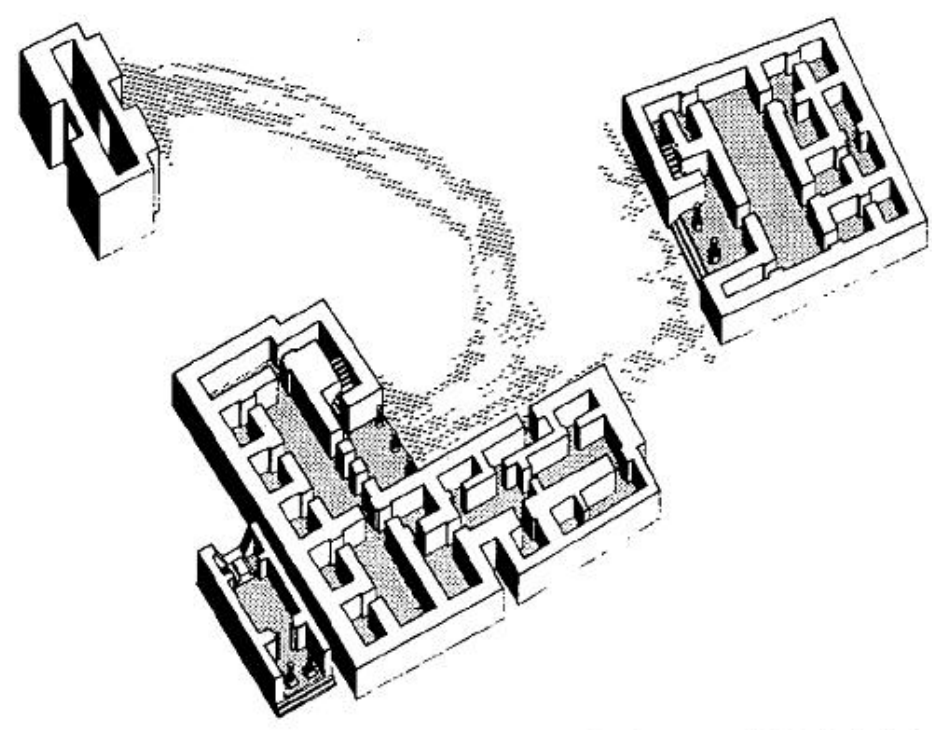

Fig. 4. Conjunto arquitectónico de Tell Taynat con Bit-hilani, s. VIII a. C. Según P. Amiet, L'Art antique du Proche-Orient. Paris, 1977, 483.

También es común en palacios y templos los accesos por eje acodado, siendo necesario dar varios giros hasta llegar en presencia del asiento del monarca o de la divinidad. ${ }^{15}$

Tampoco el material griego aparecido en el yacimiento de Cancho Roano puede ser utilizado en apoyo de esta hipótesis no sólo por su desfase cronológico, ya que corresponde al momento final del monumento, sino también por su escasa representatividad en el conjunto de hallazgos Si bien el número de vasos áticos es importante, la mayoría son sólo kylikes, 117 piezas inventariadas, y tres skyphoi. ${ }^{16}$

No se encuentran otros tipos de vasos, frecuentes en sitios donde el predominio del comercio griego es evidente. Por el contrario, el registro de objetos atribuibles al mundo fenicio-púnico es mucho más amplio Abundan las ánforas globulares, hay algunos anforiscos de base piriforme, aríbalos y varios allabastra de pasta vítrea. Objetos de marfil y hueso con inequívoca decoración oriental. Entre ellos, dos arquetas de marfil con decoración de palmetas de cuenco y escenas de animales. Una paleta de tocador también de marfil y cachas de cuchillos, de hueso, con figuras de animales. Elementos de adorno personal del mismo origen. Un escarabeo egiptizante y un escaraboide fenicio. Un sello colgante pánico y dos dados de piedra con escenas, ambos

Jerusalén son descritos como auténticos palacios con cella o sanctorum abierto sobre un patio interior. Hdt. II. 44; Ez.40-43.

15 Kaplan 1971, 293-307, considera que este tipo de acceso era originalmente mesopotámico y que aparece en Palestina en el Bronce Medio.

16 Maluquer 1981, 317; ID. 1983, 27-39 y 40-42. 
fenicios. Joyas de oro, cornalina y pasta vítrea, de la misma procedencia. Dos placas de bronce con motivo agallonado de roseta similar a la decoración del thymiaterion fenicio de Cerro del Peñón (Málaga). ${ }^{17}$

Evidentemente, la variedad de objetos procedentes del mundo feniciopúnico indica que es con el sur, y más concretamente con las colonias fenicias, con las que tiene relación Cancho Roano. Incluso hay que considerar que la abundante cerámica ática de este paraje parece venir a través de Gadir, la cual se encarga de su distribución en todo el Sur de la Península. ${ }^{18}$

Todo ello nos hace afirmar que no existe indicio seguro para suponer que fueron los griegos quienes exportaron éste modelo de edificio. Por el contrario, parece muy verosímil que los fenicios, a los que se les puede atribuir sin problemas este tipo de obra, fueron los que posibilitaron su construcción aquí en Cancho Roano.

J. Maluquer propuso como hipótesis que este complejo fue inicialmente concebido como un santuario. Realmente, el parecido de la planta de Cancho Roano con santuarios semitas del Mediterráneo Oriental es muy significativo. ${ }^{19}$ Especialmente se parece a los templos fenicios o de su influencia de Líbano y Chipre, que generalmente imitan la planta esencial de un palacio, al considerarse, a veces, el templo como morada terrestre de la divinidad. Donde la sala de aparato se convierte en cella, el patio de acceso en lugar de ritos y las habitaciones secundarias en almacén de ofrendas y objetos para el culto.

Sin embargo, en principio hay que descartar que el edificio de Cancho Roano sea un santuario indígena de construcción local. Ninguna de las soluciones arquitectónicas en él aplicadas es autóctona. No lo es el gran paramento externo, ni los accesos por eje acodado, ni la amplitud de las techumbres, ni evidentemente el aspecto mediterráneo de sus formas constructivas, etc. Además, la organización compleja del edificio, en el caso de que fuera un lugar de culto, estaría adaptada a su uso como tal. La cella, el patio, el témenos y la forma de conjunto del propio lugar de culto tienen usos específicos relacionados con el mito, advocación y usos culturales propios de la divinidad allí supuestamente adorada, que se trataría sin duda de una divinidad semita. ${ }^{20}$

17 Maluquer 1981, 324, 339-340, 288, 342-343, 346, 347, 349-362; ID. 1983, 108-109, 111-112, 91, 95, BLÁZQUEZ 1984, 127-139.

18 Fernández Jurado $(1985,65)$, se aproxima a este problema cuando dice: "El comercio ampuritano dominó el Levante español, encontrándose también sus productos en el interior, como es el caso de Zalamea de La Serena, en Badajoz, donde se documenta el mismo horizonte de siglo V a.C. que en Huelva, con copas Castulo y copas con decoración de hojas de olivo. Maluquer, desconocedor del caso de Huelva en el momento de redactar sus trabajos, propone rutas terrestres para estos materiales, pero es evidente que hoy por hoy no hay que descartar una ruta a través de Huelva".

19 Estructuras sacras con la organización o elementos que aparecen en Cancho Roano se conocen especialmente en Fenicia y Chipre. En Sarepta (Líbano) el templo de Astarté tiene una organización palacial similar PRITCHARD 1973, 91-92. En Chipre son también varios los santuarios con articulación palacial. como el de Voni, al sur de Khytera, o el hallado en Politiko (Tamassos) y el de Meniko-Litharkes, entre otros. Gjerstad 1937, 94; Karageorghis 1982, 291; 1968, 201; Al-Radi 1983, 78. Pero quizá donde la relación sea más patente es con los santuarios fenicios de Kition, la vieja colonia tiria. Sobre dichos santuarios véase, entre otros, Karageorghis 1981, 993-994; ID. 1982, 722-725; DusSAud 1950, 77-78; Dupont-Sommer 1973, vol. 1, 287-292.

20 Habría que convenir qué la población local había recibido tal cúmulo de influencias religiosas orientales, como para que este supuesto santuario de uso indígena no difiriera de uno oriental. Evidentemente, el supuesto 


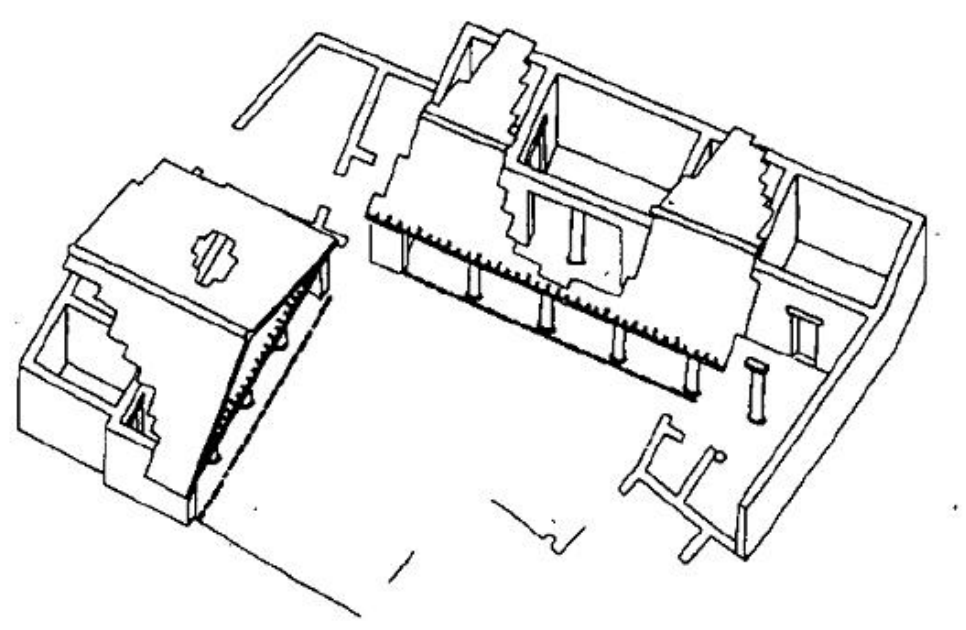

Fig. 5. Acquarossa. Ensayo de reconstrucción del edificio orientalizante. Según M. Stradberg Olofsson et alii: “Acquarossa”, en S. Stopponi (ed.), Case e palazzi, Milán, 1985, 56, fig. 9.

¿Pero realmente estamos ante la planta de un santuario, o tal edificio es susceptible de tener otra finalidad?

Ya hemos visto que santuarios fenicios reproducen la planta palacial del Próximo Oriente en sus elementos básicos. En esta línea el complejo de Cancho Roano podría ser considerado como tal.

Tampoco un vistazo a otras áreas del Mediterráneo Occidental, donde hay construcciones relativamente similares en medios indígenas, nos ha aclarado la problemática del edificio de Cancho Roano.

En Etruria, tres edificios de notable influencia oriental, equiparados a palacios y Bitt-hilani, han sido y siguen siendo motivo de polémica sobre su función. Son los de Acquarossa, Poggio Civitate (Murlo) y Montetosto.

El edificio orientalizante de Acquarossa está estructurado, no se sabe a ciencia cierta si en cuatro alas pertenecientes a una estructura de peristilo, o bien con tres alas subordinadas a una cuarta. Es un complejo monumental donde las terracotas arquitectónicas halladas indicarían que se trata de un edificio de función religiosa. Para diversos autores, la estructura corresponde a la de un palacio, sin llegar, según algunos, a excluir una actividad religiosa (fig. 5). ${ }^{21}$

Más problemática aún es la interpretación de los complejos orientalizante y arcaico de Poggio Civitate (Murlo).

no es sostenible, pues ni siquiera se ha podido demostrar que las estatuillas y objetos de culto orientales o de esta influencia hallados en la Península Ibérica, sean identificados por los indígenas con su significado original. Por ello difícilmente se puede haber llegado al estadio tan avanzado de implantación de un culto o ritual fenicio en Extremadura. Véase a este respecto: Alvar 1991, 351-356.

21 Stradberg Olofsson et alii 1985, 57. 

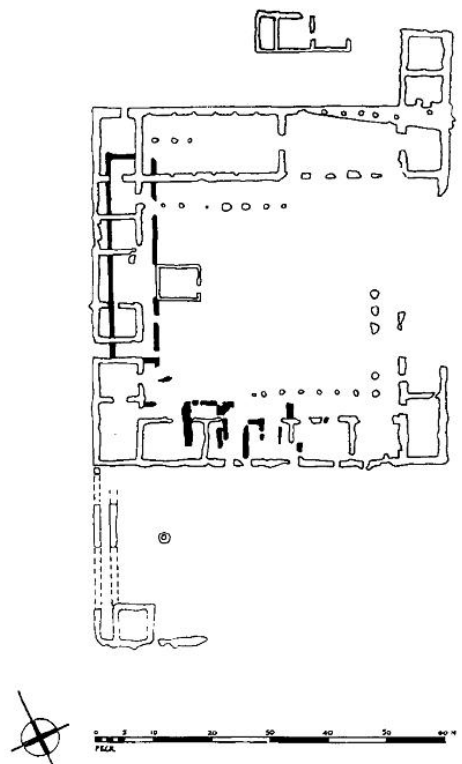

Fig. 6. Poggio Civitate. Edificio orientalizante (en negro) y estructura arcaica. Según E. O. Nielsen; K. M. Philips: "Poggio Civitate (Murlo)", en S. Stopponi, Case e palazzi, Milán, 1985, 65, fig. 2.

El modelo constructivo en ambas fases es, sin duda, oriental. La primera construcción fue alzada en torno al 650 a.C. Un incendio permitió la construcción de un nuevo edificio entre el 600 y 590 a.C. Cincuenta años después, entre 550 y 530 a.C., enigmáticamente, el edificio es demolido y ritualmente soterrado, incluso con sus propias terracotas arquitectónicas (fig. 6).

Inicialmente fue considerado como un templo, después se abrió paso la creencia de que era un palacio, siendo considerado por algunos como sede de una liga de pueblos, de mayor o menor envergadura según los autores. Explicación que no cuenta con ninguna base sólida, sólo las dificultades para considerarlo tempo o palacio, y el hecho de encontrarse Poggio Civitate a equidistancia de Volterra, Areno, Chiusi, Roselle y Vetulonia. ${ }^{22}$

Completa la controversia el edificio similar de Montetosto. Se trata también de una construcción cuadrada en torno a un patio. Este se halla en la llanura, en el camino que une Caere con su puerto, Pyrgi.

El edificio se data entre el 530 y 520 a.C. y es considerado como un santuario, aunque los argumentos no me parecen totalmente convincentes. Ejemplo único, por ahora, según G. Colonna, de santuario en forma de palacio en Etruria. ${ }^{23}$

22 NiElsen - Phillips 1985, 64-9.

23 Colonna 1985, 192-195. 
Los complejos etruscos de Acquarossa, Poggio Civitate y Montetosto sirven, pues, para considerar que el hallazgo de este tipo de edificio en medios no semitas no es un fenómeno aislado. Sin embargo, la propia controversia desatada sobre el uso de cada uno de ellos no contribuye a aclarar, aunque sea marginalmente, la finalidad asignada a la obra extremeña.

\section{Un edificio vinculado a la actividad comercial fenicia}

Algunas de las evidencias arqueológicas nos permiten afirmar que tal edificio está estrechamente ligado a la actividad mercantil fenicia en la zona. En la construcción se encuentran rastros de su utilización como centro comercial.

El hallazgo de un depósito de ánforas fenicias del siglo VI a.C. en una de las habitaciones es un primer indicio, que nos señala la traída de algún alimento elaborado de la zona del Estrecho.

También en el edificio apareció, a nivel del piso, una pepita de oro. En una de las habitaciones, el suelo estaba embebido de mercurio, el cual se utilizaba, sin duda, para eliminar las impurezas del oro. Procedía seguramente de Almadén, donde se extraía el cinabrio ya en la Antigüedad y cuyo nombre antiguo, Sisapon, es sin duda fenicio. ${ }^{24}$ Allí también han aparecido restos de balanzas y pesos de bronce, la mayoría de valores pequeños. Dos de aproximadamente 31 gramos, otros dos doblan esa cantidad, otro la multiplica por diez, pesa 304,76 gramos y uno pesa $145,99 .{ }^{25}$ Sin duda, en razón de su reducido peso, son para pesar metales preciosos.

Sin duda, el de Cancho Roano es un edificio donde se hacían transacciones comerciales, siendo los metales preciosos la contrapartida de los objetos foráneos importados. ${ }^{26}$

La capacidad de adquisición de productos exóticos señalada por los ricos hallazgos de Cancho Roano no se puede interpretar de otro modo que por la oferta de algo, los metales preciosos, en abundancia y dentro de una red comercial muy fluida. El hallazgo de importaciones en la cantidad que ha aparecido en Cancho Roano es, sin duda, el reflejo casi exacto de exportaciones parangonables. ${ }^{27}$

La posición de Cancho Roano parece ser especialmente indicada en relación con esta actividad comercial. Como hemos podido constatar, el monumento se encuentra en el extremo sur del conjunto de yacimientos de plomo argentífero de Castuera, con-

24 Maluquer 1981, 285-286; DuÇAND 1980 105, n. 219, considera que es un nombre claramente feniciooccidental: existen varios yacimientos de mercurio en la región de Almadén explotados en la Antigüedad. SiLLIÈRES 1980, 49-60.

25 Esta información no aparece en las dos memorias de excavación, sino en un trabajo posterior: MALUQUER $1985,21-22$.

26 Para Cancho Roano, véase: Maluquer 1981; 1983; BlÁzquez 1984, 127-139; Maluquer 1985, $13-14$.

27 La valía de un comerciante se debía medir por su sagacidad en proveer precisamente cosas de indudable atractivo para el indígena con quien comerciaba, que repercutían evidentemente en la cantidad de lingotes de metal que recibía a cambio o en la influencia para obtenerlos y transportarlos con seguridad. Poco les interesaban otras materias, sobre todo las de poco valor y muy voluminosas, ni inundar el país con sus productos, cuyo valor así se depreciaba. 


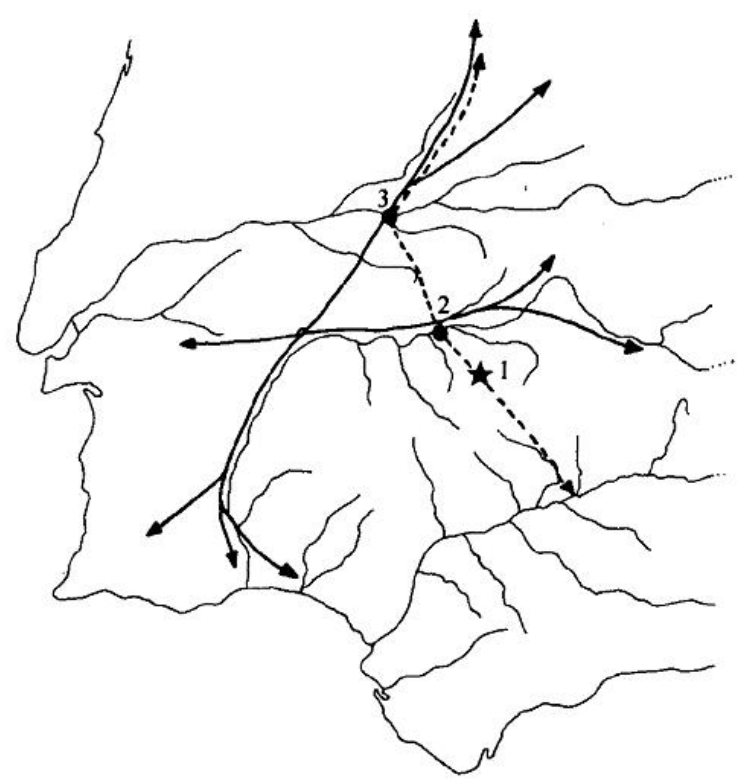

Fig. 7. Cancho Roano (1) en las vías fundamentales $S N$ del cuadrante Suroccidental de la Península Ibérica, con los vados de Medellín (2) y Alconetar (3). A partir de dos mapas de A. Alvarez Rojas; J. Gil Montes, "Aproximación al estudio de las vías de comunicación en el primer milenio antes de Cristo en Extremadura", Trabajos de Prehistoria 45, 1988, figs. 4 y 5.

junto formado al menos por 23 yacimientos. ${ }^{28}$ También se encuentra muy cerca y en el camino hacia el sur del oro aluvial de los ríos Jerte, Alagón y Arrago.

La situación de Cancho Roano en el contexto de las vías comerciales en la zona es también muy significativa y empieza a clarificarse. ${ }^{29}$

A través de la topografía y de los hallazgos de objetos orientalizantes, así como de la posición de los poblados indígenas, A. Âlvarez Rojas y J. Gil Montes han señalado para la primera mitad del primer milenio a.C. una vía de comunicación fundamental que coincide prácticamente con la "Falla de Plasencia", La cual une la desembocadura del Guadiana y la zona de Huelva con la Alta Extremadura y la Meseta, El primer accidente que franquear es la sierra de San Pedro, con el paso de Puertollano, muy cerca de La Aliseda. El siguiente paso destacable es el vado de Alconétar para atravesar el Tajo. A la altura de Plasencia la vía se bifurca, un ramal sigue el rectilíneo valle del Jerte hasta el puerto de Tornavacas, que comunica el Sur con Ávila. El otro ramal flanquea el puerto de Béjar, para adentrarse en Salamanca (fig. 7).

28 Sacado del mapa metalogénico de España, recogido por Almagro GorbeA 1977, 6-7 y 9.

29 Una primera aproximación global nos la presenta ONGIL VALENTín 1985, 327-334; un estudio más amplio y ceñido a esta cuestión es el de: Álvarez Rojas - Gil Montes 1988, 305-316. 
En dicha falla se sitúan bastantes yacimientos de oro y casiterita que vinculan toda la vía con la extracción de mineral. ${ }^{30}$

En resumen, como dicen A. Álvarez Rojas y J. Gil Montes “... (Esta) gran vía natural noroeste-suroeste une las regiones del suroeste de Portugal y la zona de Huelva con la Meseta Norte, utilizando dos elementos geográficos fundamentales: El río Guadiana y la falla de Plasencia, con un alto grado de rapidez en la comunicación y con mínimo esfuerzo, dada su gran rectitud y pequeñas altitudes". ${ }^{31}$

Esta vía parece abastecer a los fenicios de los metales obtenidos en ella misma y más al norte, a través de Huelva y la desembocadura del Guadiana, seguramente controlado dicho comercio por las gentes allí asentadas. Esta deja de ser vía exclusiva o casi exclusiva en un momento que los autores citados sitúan en el 600 a.C. Una segunda vía sustituye o entronca con la anterior, desde el Guadalquivir a la altura de Córdoba, por Cancho Roano, pasando el Guadiana por el vado de Medellín y entroncando con la vía anterior por el vado de Alconétar en el Tajo. ${ }^{32}$

En mi opinión, dicho cambio de vía está ligado a la problemática de relaciones entre los fenicios de Gadir y los tartesios, que parecen pasar por un período de ruptura en este momento. También con el cambio de horizonte cultural que se aprecia en las colonias fenicias occidentales en el siglo VI a.C. ${ }^{33}$

Mi impresión es que la caída momentánea del comercio de metales por los problemas en el Mediterráneo Oriental y concretamente en Fenicia, provocó el colapso del "mercado productor". La infraestructura socioeconómica tartésica, que era muy dependiente de dicho comercio, debió tambalearse, especialmente en lo que se refiere a su forma de jefatura. Todo ello provocó consecuentemente la crisis de sus relaciones con los fenicios de Gadir. Se debió restablecer la demanda de metales, momentáneamente hundida, pero los fenicios no podían contar ya con la infraestructura tartésica que hacía llegar hasta la costa los metales del interior.

En este contexto, la apertura de la ruta fenicia desde el Guadalquivir por Cancho Roano y Medellín parece suplantar la vía anterior, controlada por los indígenas. ${ }^{34}$

En esta vía, Cancho Roano es un hito importante, a la vez que seguramente drenaba los recursos de plata de su entorno.

Por otro lado, en el mismo Medellín, a pocos kilómetros al norte de Cancho Roano, se han hallado algunos fragmentos de cerámica local con grafitti fenicios que no deja lugar a dudas sobre la existencia de agentes fenicios allí, en torno al 600 a.C. ${ }^{35}$

30 Álvarez Rojas - Gil Montes 1988, 308-309 y fig. 1.

31 Álvarez Rojas - Gil Montes 1988, 313.

32 Álvarez Rojas - Gil Montes 1988, 315.

33 M. E. Aubet, muy amablemente, me ha señalado que ha podido documentar cambios en esta fecha en sus últimas excavaciones en la desembocadura del Guadalhorce (Málaga). Arcila, 9 de noviembre de 1989.

34 El Guadalquivir se configura como la gran vía de penetración con dos ramales, uno, el extremeño, y otro, siguiendo el río, hasta Sierra Morena, donde Cástulo será el gran centro comercial. Camino jalonado también de ricas manufacturas importadas de los siglos VII y VI a.C. BISI 1983, 121; AUBET 1987, 249.

35 Así lo afirma Almagro Gorbea 1977, 274; vuelve a incidir sobre ello en Almagro Gorbea 1983, 446, n. 112; también en el yacimiento de la Peña Negra en Alicante, a 25 kilómetros a vista de pájaro de la costa se ha podido documentar la presencia de fenicios en el poblado indígena. Un grafito con el nombre BDSMN en la base de un plato de barniz rojo de factura local así lo demuestra (GonZález Prats 1986, 270-273). 
Un texto de Justino es elocuente sobre la presencia de comerciantes extranjeros en el medio indígena. Según relata, el rey Argantonio invita a unos focenses que han llegado a su territorio a quedarse como inquilini. ${ }^{36}$ Sin duda como comerciantes instalados, no transeúntes, lo cual permitiría al rey obtener ventajas de la competencia que se estableciera entre fenicios y griegos. Situación a la que no estaban dispuestos seguramente los fenicios de Cádiz. Este es un dato más que avala el interés de los fenicios por tener una cabeza de puente en los mercados próximos a los lugares de extracción y no dejar que otros lo hicieran. Dicho texto parece tener relación también con lo que comentamos antes de la crisis de relaciones entre Gadir y Tartessos.

El comportamiento comercial fenicio-púnico se caracteriza por acercarse lo más posible al mercado más próximo al lugar de extracción de los recursos que le interesan, con el fin de eliminar al máximo cualquier añadido de valor sobre éstos. El motivo no es obtener un beneficio por la simple acaparación del mayor tramo posible de transporte, sino conseguir poco valorado el producto o productos que quiere obtener, debido a su abundancia en el mercado próximo al lugar de extracción, y a cambio aportará productos exóticos raros allí y por ello altamente preciados. ${ }^{37}$

Esta tendencia hacia la captación de los "mercados de origen" hace que la organización de las entidades comerciales sea muy compleja y se tienda a establecer oligopolios muy capitalizados para maximalizar los beneficios.

Documentada la implicación comercial del edificio de Cancho Roano, también su ubicación en una vía comercial y su relación con el mundo fenicio, nos resta desentrañar su implicación concreta en este contexto.

Por lo expresado en páginas atrás, creemos que dos hipótesis se pueden traer a colación. Una, la suposición de que se trata de un edificio diseñado por fenicios, pero ocupado por algún régulo indígena. $\mathrm{O}$ en otro caso, de un edificio diseñado por fenicios y usado y dirigido por ellos.

En el supuesto de que se trate de un edificio utilizado por los fenicios, evidentemente, hay que desechar su función palacial y considerarlo un edificio mercantil-sacro, cuyo paralelo estaría en los almacenes de Al Mina, a los cuales J. Maluquer hizo referencia. En apariencia una extraña combinación pero como veremos no es un caso único, e incluso podemos afirmar que se trata de una práctica usual en la expansión tiria, vincular lo sagrado con el comercio.

Desde el primer momento existe un estrecho lazo entré la divinidad tutelar de Tiro, Me/kan, y la empresa comercial de esta ciudad. Es el propio oráculo de su templo el que propicia la instalación de un lugar sacro y encubiertamente el inicio de las transacciones económicas en el Extremo Occidente en fecha remota, como se deduce del relato de la fundación de Gadir. ${ }^{38}$

36 Just. XLVIII, 4.4; texto similar en Hdt. I, 163; sobre esta condición de inquilini, véase: Morel 1983, 569.

37 Este comportamiento comercial se ha podido documentar claramente en Cerdeña, donde se ha podido apreciar cómo los fenicios se ocuparon de tener un atento control no sólo de la costa. sino también de las vías de acceso a los centros mineros del interior de la isla. "El conjunto de los testimonios sobre la irradiación fenicia de época arcaica en Cerdeña documenta la acción de un verdadero y propio diseño estratégico. vuelto no a la ocupación física del territorio, sino a la obtención de sus recursos" (BONDI 1983, 85).

38 Str. III. $5,5$. 
Así, cuando la actividad mercantil se convierte en la fuente principal de recursos de una comunidad, como en el caso de la tiria, el santuario de su dios principal, Melkart, se convertirá en el principal valedor de esta actividad y el promotor de la empresa colonial. Dicha vinculación se puede apreciar muy bien en los enfrentamientos de la facción mercantil en torno a Elisa y el templo de Melkart y la facción terrateniente en torno a Pigmalión, enfrentamientos que condujeron a la fundación de Carthago. ${ }^{39}$

Esta estrecha relación queda reflejada en las factorías y colonias fenicias occidentales, en el carácter primigenio y en la importancia de algunos de sus santuarios. Tanto es así que en los relatos sobre la fundación de Gadir, Utica y Lixus, consideradas las más antiguas, la referencia a su antigüedad les viene dada por sus santuarios y no por el hábitat. ${ }^{40}$

Ejemplo material ilustrativo de ello es Mogador. En este islote, a pesar de la provisionalidad de la ocupación de la factoría, en tiendas o chamizos, el único elemento pétreo es un betilo de 1,47 metros de altura, que señala la instalación de un recinto sagrado aquí. ${ }^{41}$

Por otro lado, se puede documentar la práctica de instalar un santuario en un núcleo o mercado indígena donde los fenicios van a comerciar de forma prolongada.

En Rodas, famosa por sus manufacturas en bronce, Kadmos, según nos relata Diodoro, consagró un témenos a Poseidón, con el pretexto de haber sufrido una tempestad, y dejó allí algunos fenicios para que lo cuidaran. ${ }^{42}$

Lo mismo hacen los fenicios cuando llegan a Thasos, pero allí, según nos cuenta Herodoto, lo que fundan es un templo a Herakles, dejando también allí algunos fenicios. Aquí la presencia fenicia se vinculaba a la explotación de las minas de oro de la isla. ${ }^{43}$

En el Mediterráneo occidental vemos los mismos planteamientos. Santuarios fenicios que se instalan en núcleos indígenas relacionados con la extracción minera y centros artesanales o mercantiles importantes.

Un documento excepcional de cómo se realizaba este tipo de fundación sacra nos lo aporta un santuario de Pyrgi. Se trata de unas láminas de oro con dos textos, uno en etrusco y otro en fenicio o púnico, procedentes de Pyrgi, puerto del estado etrusco de Caere. ${ }^{44}$ En ella se dice que el rey o magistrado principal (Zilath) dedica un lugar sagrado, templo o cella, a la diosa Astarté. Ello ocurría a fines del siglo VI a.C. o comienzos del V a.C. ${ }^{45}$

\footnotetext{
39 Véase Alvar - GonZÁlez WaGner 1985, 79-95.

40 Véase BunNens 1979, 186.

41 Sobre el betilo, véase: Jodin 1966, 52; Moscati 1988, 288.

42 Diod. V, 58, 2; este texto recuerda, a su vez, lo relatado en la estela de Nora.

43 Hdt. II, 44; según G. Bunnens, la información de Herodoto procede de una tradición local, pues éste afirma haber visitado el santuario (BunNENS 1979, 112).

44 Sobre el origen exacto del texto semita no hay acuerdo, aunque parece más probable que sea púnico. A este respecto, véase: GARBINI 1965, 35-52.

45 Sobre el significado de Zilath véase: Pallottino 1955-1956, 45-72 (=1979, 754-758); en lo concerniente al hallazgo y al santuario, véase: PALLOTTINO $1964,558-563$ y 76-117 (=1979, 633-671).
} 
A través de este documento podemos apreciar cómo existía una confluencia de intereses de los comerciantes fenicio-púnicos y las autoridades locales que favorecieron la instalación de este lugar sagrado.

Vemos, pues, cómo elevar un lugar sacro en un hábitat indígena era una práctica frecuente en la empresa colonial fenicia en todo el Mediterráneo. Y parece que fue una pieza fundamental de la misma, a semejanza del importante papel económico del templo en el Próximo Oriente, a partir de cuya estructura evolucionó el santuario fenicio.

Las razones que justifican una construcción de este tipo en un medio indígena en relación con el comercio son múltiples.

Suponemos que la apertura de un lugar sacro, la petición de permiso para levantar un ara y realizar un sacrificio, debía ser bien visto por los indígenas. Presentar una imagen piadosa, dar a entender que estaban allí por un mandato de un dios propio, etc., permitía con mayor facilidad la apertura de intercambios en mercados de comunidades organizadas. El ara y el sencillo témenos construido, o el santuario indígena considerado asiento de la divinidad fenicia, se convirtieron en lugares donde se cerraban las transacciones con la garantía divina. ${ }^{46}$

El nuevo lugar sacro se convertirá en una sucursal del santuario que ha propiciado la empresa.

Pero los paralelos más exactos de lo que pudo ser el edificio de Cancho Roano en este supuesto de santuario lo encontramos en una inscripción de los Heracleístas tirios de Delos y en el edificio de los Poseidoniastas de Beirut.

En Delos, una inscripción del siglo II a.C. nos habla de una asociación de Heracleístas tirios que solicita permiso a las autoridades atenienses, en ese momento dueñas de la isla, para construir un témenos a Herakles tirio (Melkart). La asamblea de la asociación está formada por armadores y negociantes de Tiro asentados en Delos, y la misma tiene lugar en el templo de Apolo. La finalidad de la asociación y de la construcción del témenos es salvaguardar los intereses económicos y sociales de sus miembros y crear contactos fructíferos con los habitantes de la isla. ${ }^{47}$

En Beirut, las excavaciones del santuario y sede de la asociación de los Poseidoniastas han mostrado a la luz, entre las habitaciones de culto, otras destinadas a almacenes y a hospedaje. ${ }^{48}$

La configuración del edificio de Cancho Roano puede responder a los modelos precedentemente descritos. Un complejo mercantil fenicio, con sus almacenes para mercancías traídas del sur, dependencias donde pesar y depurar el metal precioso y una sala sacra, la cámara central consagrada a una divinidad fenicia, todo ello rodeado de paramentos sólidos de protección.

A esta interpretación naturalmente se le pueden hacer objeciones. Quizá la más grave sea que no se ha encontrado ningún rasgo definitivo de su función cultual, re-

46 G. Bunnens explica así la instalación de santuarios en medio indígena, como la solución para las suspicacias mutuas entre autóctonos y fenicios cuando se iba a establecer una relación larga de intercambios. Era el lugar donde las partes contratantes se reunían (BunNENs 1979, 284).

47 Bonnet 1988, 373.

48 Bonnet 1988, 373, n. 1. 
ceptáculo del árbol sagrado, objetos exclusivos de culto, etc. Aunque se suponga la existencia de un ara en la terraza superior.

También encontramos un mayor parecido de este edificio, por su sólida y maciza construcción, con los hilani de la zona de Siria de la primera mitad del primer milenio, más que con los santuarios, arquitectónicamente más débiles y con menos unidad.

Se abre paso pues la otra posibilidad, un edificio diseñado por fenicios, pero ocupado por algún régulo indígena. Lo cual nos permite explicar más fácilmente la función de la cámara principal, más profunda y sin puertas a las otras habitaciones, que ocupa el lugar de lo que debía ser la sala de representación o de aparato en el palacio, o la cella en el templo y que no puede ser interpretado como tal. En realidad puede ser el thesaurus, lugar protegido del edificio palacial, que precisamente por ello no tiene un acceso fácil. ${ }^{49}$

Esta construcción sería seguramente una de las máximas expresiones de regalo inmueble fenicio a un jefe local, a semejanza de lo que pudo ser el tesoro de La Aliseda entre los regalos muebles. ${ }^{50}$

La construcción maciza, la cámara central protegida y la parquedad de habitaciones, obligan a pensar que no se trata propiamente de un palacio para un príncipe indígena, sino que su forma parece estar perfectamente adaptada a lo que se requiere de él. El almacenamiento de ánforas en una de las dependencias, el mercadeo de metales allí desarrollado, su ubicación precisa, etc., denotan que el edificio ha sido construido para que un príncipe con función redistributiva reúna en él las riquezas metalíferas de la zona y la que es traída desde el norte.

Sería, por tanto, el edificio de Cancho Roano la muestra palpable de un comercio "de tratado" con jefes indígenas. Forma de comercio necesaria para asegurar, por un lado, la transitabilidad de las mercancías y, por otro, como forma para dinamizar a través de dicha jefatura "redistributiva" la producción metalífera de la zona para ser adquirida o toda o en parte por los fenicios. ${ }^{51}$

En resumen, entendemos que las dos posibilidades expuestas, construcción palacial de jefe indígena, o santuario-lugar de comercio fenicio, nos parecen válidas para explicarla existencia de este "extraño" edificio en tan insólito paraje. Aunque nos parece que la primera, construcción palacial, es más verosímil.

Por otro lado, creemos haber demostrado la firme implicación de este edificio con la actividad comercial y las formas en las que ésta se manifiesta.

A través de las actuales excavaciones, esperamos que se despeje la interrogante del espacio de ocupación concreto en el que se ubica el monumento, o bien un hábitat, o mejor un mercado con pocas construcciones fijas. Esperamos también que sea posible definir su conexión con Medellín, el lugar fuerte en el vado de paso del Guadiana,

\footnotetext{
49 Debo a J. Martínez-Pinna la sugerencia de que esta habitación pueda ser un thesaurus.

50 A pesar de que la mano de obra pudiera ser indígena, la depurada concepción del edificio y las nociones técnicas en él aplicadas, señalan el trabajo de un arquitecto no local.

51 Sobre sistemas de jefaturas en la Península Ibérica pueden ser ilustrativos los trabajos siguientes: Nocete Calvo 1984, 289-304; Alvar 1986, 161-175.
} 
Seguramente lo excepcional de este tipo de jefatura "redistributiva" en la zona y la rareza de la construcción a ella asociada hace que el monumento dure poco en su uso original, seguramente menos de un siglo, convirtiéndose en un quemadero de cadáveres de un ritual funerario autóctono.

\section{Bibliografía}

Almagro Gorbea, M.

(1977): El Bronce Final y el periodo orientalizante en Extremadura, Madrid.

(1983): "Colonizzazione e acculturazione nella Penisola Iberica", en Modes de contacts et processus de transformation dans les sociétés anciennes, Pisa-Roma, 429-461.

Al-Radi, S. (1983): Phlamoudhi Vounari: a Sanctuary Site in Cyprus (=Studies in Mediterranean Archaeology 65), Götenborg.

Alvar EzQuerra, J.

(1986): "Theron, rex Hispaniae citerioris (Macr. Sat., I, 20, 12)”, Gerión 4, 161 175.

(1991): "La religión como índice de aculturación. El caso de Tartessos", [en] II Congreso Internazionale di Studi Fenici e Punici, Roma, 351-356.

Alvar Ezquerra, J. - González Wagner, C. (1985): "Consideraciones históricas sobre la fundación de Cartago", Gerión 3, 79-95.

Álvarez Rojas, A. - Gil Montes, J. (1988): “Aproximación al estudio de las vías de comunicación en el primer milenio antes de Cristo en Extremadura", TP 45, 305316.

Амiet, P. (1977): L'art antique du Proche-Orient, Paris.

Aubet, M. E. (1987): Tiro y las colonias fenicias de Occidente, Barcelona.

Bisi, A. M. (1983): "L'Espansione fenicia in Spagna”, [en] Convegno sul tema: Fenici e Arabi nel Medizerraneo, Roma, 97-151.

Blanco Freijeiro, A. (1982): "El enigma de Cancho Roano", Investigación y Ciencia, enero de 1982, 42-43.

Blázquez, J. M. (1984): "Marfiles fenicios de Cancho Roano (Badajoz) con el árbol de la vida y sus prototipos", Boletín de la Asociación Española de Orientalistas 20, 127-139.

Bond, S. F. (1983): "L’Espansione fenicia in Italia", [en] Convegno sul lema: Fenici e Arabi nel Mediterraneo, Roma, 63-95.

Bonnet, C. (1988): Melqart. Cultes et mythes de l'Heracles Tyrien en Méditerranée, Leuven.

Bunnens, G. (1979): L'Expansion phénicienne en Méditerranée, Bruselas-Roma.

Colonna, G. (1985): "II santuario di Montetosto", [en] S. Stopponi (ed.), Case e palazzi, Milán, 192-195. 
DuÇAND, J. E. (1985): “À propos de Salamis, essai de rapide mise à jour de quelquesuns des points traités par l'auteur en son 'Chypre et Canaan' (Nice, 1973)”, [en] Salamine de Chvpre. Histoire e Archéologie, Paris, 85-109.

Du Plat Taylor, J. (1956): "The Cypriot and Syrian Pottery from Al Mina. Syria”, Iraq 21, 62-92.

DuPONT-SOMmer, A. (1973): "Un anthroponyme anatolien dans une inscription phénicienne archaïque récemment trouvée à Kition en Chypre", [en] The Proceeding of the Xth International Congress of Classical Archeology, Ankara, vol. 1, 287-292.

Dussaud, R. (1950): "Kinyras. Étude sur les anciens cultes chypriotes", Syria 27, 57-81.

Fernández JuRADO, J. (1985): La presencia griega arcaica en Huelva, Huelva.

Garbini, G. (1965): “Considerazioni sull'iscrizione púnica di Pyrgi, con un contributo di G. Levi Della Vida", Oriens Antiquus 4, 35-52.

GJerstad, O. E. (1937): The Swedish Cyprus Expedition, III, Estocolmo.

GonzÁlez Prats, A. (1986): Estudio arqueológico del poblamiento antiguo de la Sierra de Crevillente (Alicante), Alcoy.

Graham, A. I. (1983): “The Historical lnterpretation of Al Mina”, Dialogues d'Histoire Ancienne 12, 51-65.

Jodin, A. (1966): Mogador comptoir phénicien du Maroc atlantique, Tánger.

Kaplan, J. (1971): "Mesopotamian Elemenis in the Middle Bronce II Culture of Palestine", JNES 30, 293-307.

KARAGHEORGIS, V.

(1981): "Chronique des fouilles et découvertes archéologiques á Chypre en 1980". BCH 105, 967-1024.

(1982): Cyprus, from the Stone Age to the Romans, Londres.

(1982a): “Chronique des fouilles à Chypre en 1981”, BCH 106/2, 685-744.

MALUQueR, J.

(1981): El santuario protohistórico de Zalamea de La Serena. Badajoz. I, Barcelona.

(1983): El Santuario protohistórico de Zalamea de La Serena. Badajoz. II, Barcelona.

(1985): "Notes sobre les relacions comercials entre la Conca del Guadiana i Andalusia en els darrers temps de la civilització tartèssica", Pyrenae 21, 11-22.

Morel, J.-P. (1983): "Les relations économiques dans L’Occident Grec”, [en] Modes de contacts et processus de transformation dans les sociétés anciennes, PisaRoma, 549-580.

Moscati, S. (1988): "La statuaria", en I Fenici, Milán, 284-291.

Nielsen, E. D. - Phillips, K. M. (1985): "Poggio Civitate (Murlo)", [en] S. Stopponi (ed.), Case e palazzi, Milán, 64-69. 
Nocete Calvo, F. (1984): "Jefaturas y territorio: una visión criticas", Cuadernos de Prehistoria de la Universidad de Granada 9, 289-304.

Ongil Valentín, M. I. (1985): “Aportaciones al estudio de la protohistoria extremeña", [en] Iberos. Actas de las I Jornadas sobre el Mundo Ibérico, Jaén, 327-334.

Pallottino M.

(1955-1956): "Nuovi spunti di ricerca sul tema delle magistrature etrusche", Studi Etruschi 24, 45-72 (=Saggi di Antichità 2, Roma, 1979, 754-758).

(1965): "Scavi nel Santuario etrusco di Pyrgi", Archaeologia Classica 16, 558-563 y 76-117 (=Saggi di Antichità 2, Roma, 1979, 633-671).

Parrot, A. (1967): Mission Archéologique de Mari III. Les temples d'Ishtarat et de Ninni-Zaza, París.

Pritchard, J.B. (1973): “The 1972 Excavations al Sarepta (Lebanon)”, RSF 1, 91-92.

Sillières, P. (1980): "Sisapo: Prospections et découvertes", AEspA 53, 49-60.

Stradberg Olofsson, S. et aliI (1985): “Acquarossa”, [en] S. Stopponi (ed.), Case e palazzi, Milán, 57.

Wooley, A. (1938): "Excavations at Al Mina, Sueidia”, JHS 58, 1-30. 